\title{
Proton Elastic Scattering from 58Ni and 90Zr at Intermediate Energy
}

\author{
M. Abdel El-moneim ${ }^{1}$, N. A. El-Nohy ${ }^{1}$, M. Abd El-Zaher ${ }^{2, *}$, S. I. Yoseph ${ }^{2}$ \\ ${ }^{1}$ Physics Department, Faculty of Science, Alexandria University, Alexandria, Egypt \\ ${ }^{2}$ Department of Basic and Applied Science, Faculty of Engineering, Arab Academy for Science and Technology, Alexandria, Egypt
}

\begin{abstract}
Elastic proton scattering at energies between 160 and $800 \mathrm{MeV}$ from $58 \mathrm{Ni}$ and $90 \mathrm{Zr}$ has been studied within the global Dirac optical model. In this work we calculate potential parameters which give good fits to the experimental data using parameterization code comprising differential cross section and analysing power measurements using DWUCK4. The real and imaginary potentials are well determined and behave regularly with energy. The behaviour of the real central effective potential shows the development of a "wine-bottle" shape in the transition energy region and the persistence of a small attractive potential in the nuclear surface region, even at $800 \mathrm{MeV}$.
\end{abstract}

Keywords Global Dirac, Optical Potential, DWUCK4, Elastic Scattering, Intermediate Energy

\section{Introduction}

In the last several years, a large number of experimental data, on proton elastic scattering at intermediate energies $(\sim 150 \mathrm{MeV}-1 \mathrm{GeV})$ were published. They prompted several global analyses of this scattering process in the framework of the relativistic optical model potential. The relativistic optical model potential[1-4], not discussed here in detail, is taken to be a sum of a Lorentz scalar potential and the time-like component of a vector potential. With such a relativistic potential a Dirac equation is solved numerically in order to calculate the elastic scattering observables. From the vector and scalar potentials it is possible to construct potentials which, when inserted into a Schrödinger equation, give rise to the elastic scattering. These "Schrödinger equivalent" potentials consist of central and a spin-orbit parts. Around $300 \mathrm{Mev}$, the real part of the central potential has a pronounced shape takes the form of a repulsive core surrounded by an attractive part[2,3]. This requirement for a repulsive core seems to be less obvious for light target nuclei such as ${ }^{4} \mathrm{He}[5]$.

The purpose of this work is to extend the global relativistic optical model analyses to a larger number of target nuclei, in particular heavier ones, in order to find more about the behaviour of optical model potential at different energies and for different mass numbers.

\section{Analysis}

* Corresponding author:

mohzaher400@hotmail.com (M. Abd El-Zaher)

Published online at http://journal.sapub.org/jnpp

Copyright (C) 2012 Scientific \& Academic Publishing. All Rights Reserved
We have analysed the bulk of the existing experimental data (mainly from LAMPF, IUCF and TRIUMF accelerators [8-12]) for elastic proton scattering at several energies: 160 , $180,200,280,400,, 489,500$ and $800 \mathrm{MeV}$, from two target nuclei, namely; ${ }^{58} \mathrm{Ni}$ and ${ }^{90} \mathrm{Zr}$. The experimental data consists of , in all cases, the differential cross section and analysing power measurements at many scattering angles. The sources of the experimental data referred to are given in tables 1-2. All cross sections, analysing powers, two composites of vector and scalar potentials, central potentials and spin-orbit potential have been analysed within DWUCK4[6].

As in ref.[5], we analyse the experimental data by solving the Dirac equation in which the optical potential is taken to be a function of a complex time-like component of a Lorentz vector potential $V(r)$, and a complex Lorentz scalar potential $S(r)$. We use the forms for the potentials $V(r)$ and $S(r)$ as

$$
\begin{aligned}
& V(r)=V_{v} f\left(r, r_{v 1}, a_{v 1}\right)+i W_{v} f\left(r, r_{v 2}, a_{v 2}\right) \\
& S(r)=V_{s} f\left(r, r_{s 1}, a_{s 1}\right)+i W_{s} f\left(r, r_{s 2}, a_{s 2}\right)
\end{aligned}
$$

The form factors $f(r, R, a)$ are chosen to be symmetrised Wood-Saxon functions given by

$$
f(r, R, z)=\{1+\exp (r-R) / z\}^{-1} \times\{1+\exp [-(r+R) / z]\}^{-1}
$$

The effective central and spin-orbit potentials arising in the second-order Dirac equation

$$
\left(\nabla^{2}+\left(E-V_{c}\right)^{2}-m^{2}-2 E\left[U_{c e n t}+U_{s . o} \sigma . L\right]\right) \psi(r)=0
$$

are written as

$$
\begin{gathered}
U_{\text {cent }}=\frac{1}{2 E}\left(2 E V+2 m S-V^{2}+S^{2}-2 V_{c} V+2 E U_{D}\right) \\
U_{D}=\frac{1}{2 E}\left[-\frac{1}{2 r^{2} A} \frac{d}{d r}\left(r^{2} \frac{d A}{d r}\right)+\frac{3}{4 A^{2}}\left(\frac{d A}{d r}\right)^{2}\right]
\end{gathered}
$$




$$
U_{\text {s.o }}=\frac{1}{2 E}\left[-\frac{1}{r A} \frac{d A}{d r}\right]
$$

and where

$$
A(r)=\left(m+S+E-V-V_{C}\right) /(m+E)
$$

\section{Discussion}

In tables 1 and 2 we give the parameters calculated at different energies for two target nuclei ${ }^{58} \mathrm{Ni}$ and ${ }^{90} \mathrm{Zr}$ using parameterization code for Global optical model formula developed by S. Hama and B.C. Clark[7].

First we start with a discussion of the results for the differential cross sections and the analysing powers obtained within the global approaches for ${ }^{58} \mathrm{Ni}$ and ${ }^{90} \mathrm{Zr}$ respectively. Figures. $(1-4)$ show the experimental data and the results of the global model analyses, described above.

Table 1. Calculated parameters at different energies for a target nuclei ${ }^{58} \mathrm{Ni}$ using parameterization code for Global optical model formula developed by S. Hama and B.C. Clark[7]

\begin{tabular}{|c|c|c|c|c|}
\hline \multicolumn{5}{|c|}{$58 \mathrm{Ni}(\mathrm{p}, \mathrm{p}) 58 \mathrm{Ni}$} \\
\hline Energy & 200 & 280 & 400 & 489 \\
\hline Data ref. & Ref [8] & Ref [9] & Ref [8] & Ref [9] \\
\hline Vv & 285.6813 & 265.9549 & 238.5455 & 220.0838 \\
\hline rv1 & 1.04332 & 1.034851 & 1.028085 & 1.026624 \\
\hline av1 & 0.630668 & 0.64677 & 0.648381 & 0.644384 \\
\hline Wv & -60.818 & -67.1649 & -74.632 & -79.1017 \\
\hline rv2 & 1.154619 & 1.136362 & 1.118842 & 1.110039 \\
\hline av2 & 0.499888 & 0.49736 & 0.494953 & 0.494253 \\
\hline Vs & -380.911 & -364.248 & -341.722 & -326.415 \\
\hline rs1 & 1.03848 & 1.031591 & 1.026418 & 1.025754 \\
\hline as1 & 0.661155 & 0.680141 & 0.6871 & 0.685517 \\
\hline Ws & 49.28434 & 52.19753 & 56.49159 & 59.42559 \\
\hline rs2 & 1.173112 & 1.156148 & 1.136494 & 1.125149 \\
\hline as2 & 0.417826 & 0.427245 & 0.4345 & 0.436576 \\
\hline$\sigma R$ & 708 & 709 & 717 & 724 \\
\hline
\end{tabular}

Units :energies $(\mathrm{MeV})$, lengths ( $\mathrm{fm}), \sigma \mathrm{R}$ total cross section in (mb).

Table 2. Calculated parameters at different energies for a target nuclei ${ }^{90} \mathrm{Zr}$ using parameterization code for Global optical model formula developed by S. Hama and B.C. Clark[7]

\begin{tabular}{|c|c|c|c|c|}
\hline \multicolumn{5}{|c|}{ As for table 1, except for $90 \mathrm{Zr}(\mathrm{p}, \mathrm{p}) 90 \mathrm{Zr}$} \\
\hline Energy & 160 & 180 & 500 & 800 \\
\hline Data ref. & Ref [10] & Ref [10] & Ref [11-12] & Ref [13] \\
\hline $\mathrm{V}_{\mathrm{v}}$ & 297.872 & 292.7478 & 218.9085 & 167.4277 \\
\hline $\mathrm{r}_{\mathrm{v} 1}$ & 1.072183 & 1.069205 & 1.050145 & 1.060448 \\
\hline $\mathrm{a}_{\mathrm{v} 1}$ & 0.642531 & 0.652106 & 0.671618 & 0.675695 \\
\hline $\mathrm{W}_{\mathrm{v}}$ & -57.6861 & -59.6585 & -80.7083 & -92.1467 \\
\hline $\mathrm{r}_{\mathrm{v} 2}$ & 1.177077 & 1.17057 & 1.119311 & 1.099537 \\
\hline $\mathrm{a}_{\mathrm{v} 2}$ & 0.53292 & 0.532157 & 0.525846 & 0.529356 \\
\hline $\mathrm{V}_{\mathrm{s}}$ & -393.067 & -388.558 & -327.456 & -281.511 \\
\hline $\mathrm{r}_{\mathrm{s} 1}$ & 1.067988 & 1.065545 & 1.051019 & 1.062747 \\
\hline $\mathrm{a}_{\mathrm{s} 1}$ & 0.669723 & 0.67927 & 0.709919 & 0.705043 \\
\hline $\mathrm{W}_{\mathrm{s}}$ & 48.31454 & 49.02255 & 60.57265 & 68.55155 \\
\hline $\mathrm{r}_{\mathrm{s} 2}$ & 1.192894 & 1.187695 & 1.133353 & 1.107997 \\
\hline $\mathrm{a}_{\mathrm{s} 2}$ & 0.430693 & 0.434273 & 0.455987 & 0.452686 \\
\hline$\sigma_{\mathrm{R}}$ & 991 & 989.7 & 989 & 973 \\
\hline
\end{tabular}

Units : energies $(\mathrm{MeV})$, lengths $(\mathrm{fm}), \sigma_{\mathrm{R}}$ total cross section in $(\mathrm{mb})$.

Secondly, we concentrate on the results of the potential composite analysis of vector and scalar potentials which are shown in figures. $(5-8)$.

Finally, the energy dependence for two composites of central and spin potential are shown in figures $(9-12)$.

As expected, the global fits to the differential cross sections are very good for both targets at all energies. the analysing power, however, is sensitive to the type of the applied models. There are small differences in analysing power $\left(A_{Y}\right)$ between the results of global models and experimental data for both targets. This is shown in figures 2 and 4 . No experimental data at $160 \mathrm{MeV}$ for proton scattering on zirconium is available to the best of our knowledge. For higher energies the differences are larger, the predictions differ in magnitude but have similar shapes. At larger angles, the minima and maxima in Ay are reproduced well by this model but the whole pattern is shifted towards smaller angles relative to the experimental data. In tables 1 and 2 we also give the reaction cross sections, $\sigma_{R}$, calculated for different parameter sets although experimental values were not included in the parameter calculations.
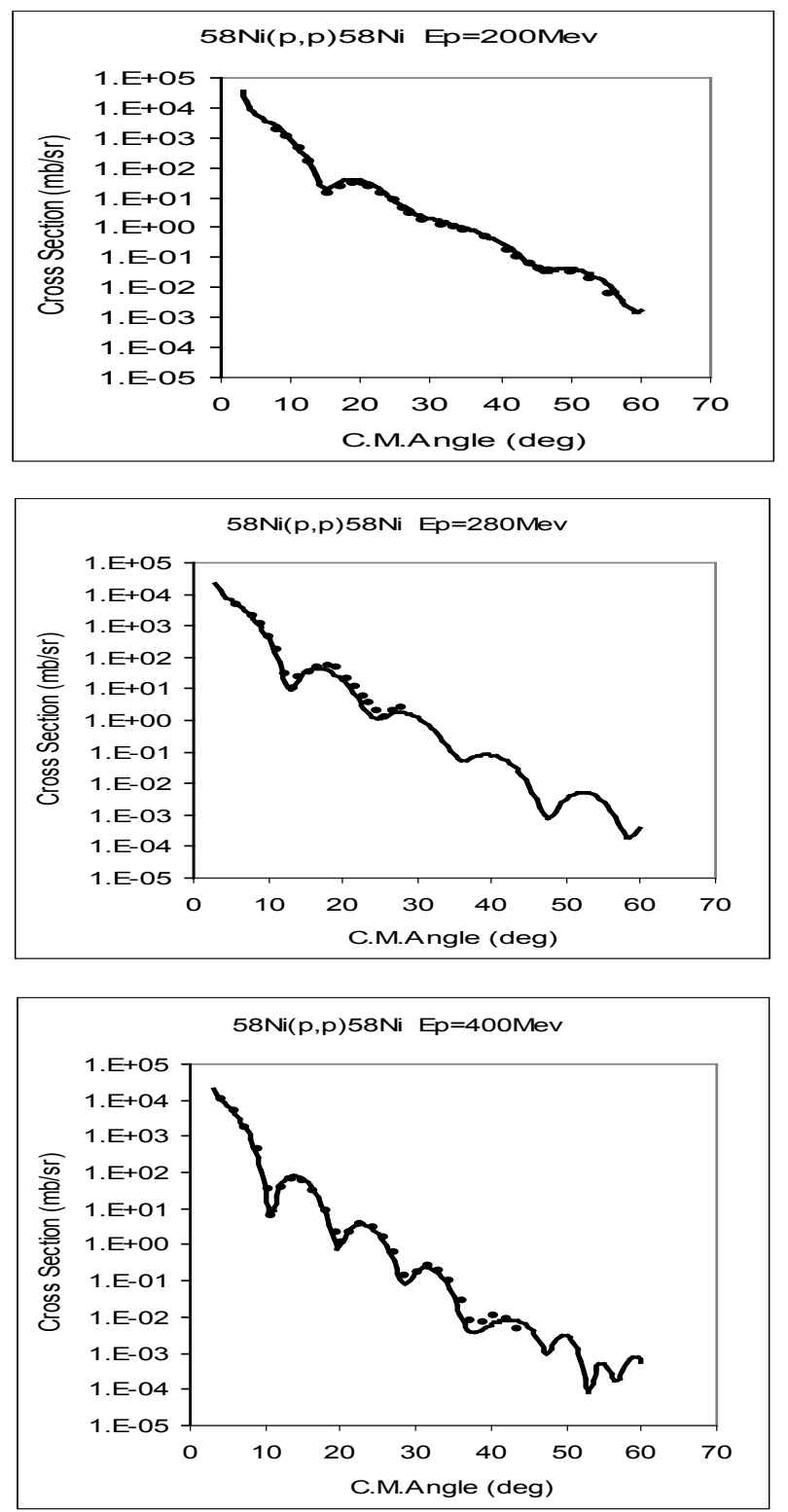
Journal of Nuclear and Particle Physics 2012, 2(1): 1-6

3

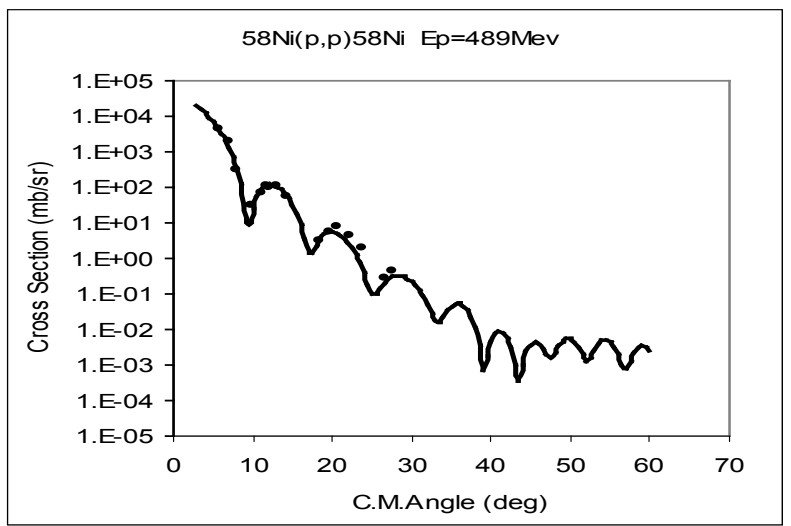

Figure 1. The differential cross section for $\mathrm{p}+58 \mathrm{Ni}$ elastic scattering at $200,280,400$ and $489 \mathrm{MeV}$. The solid curves represent the global potential calculations; the solid circles represent the experimental data
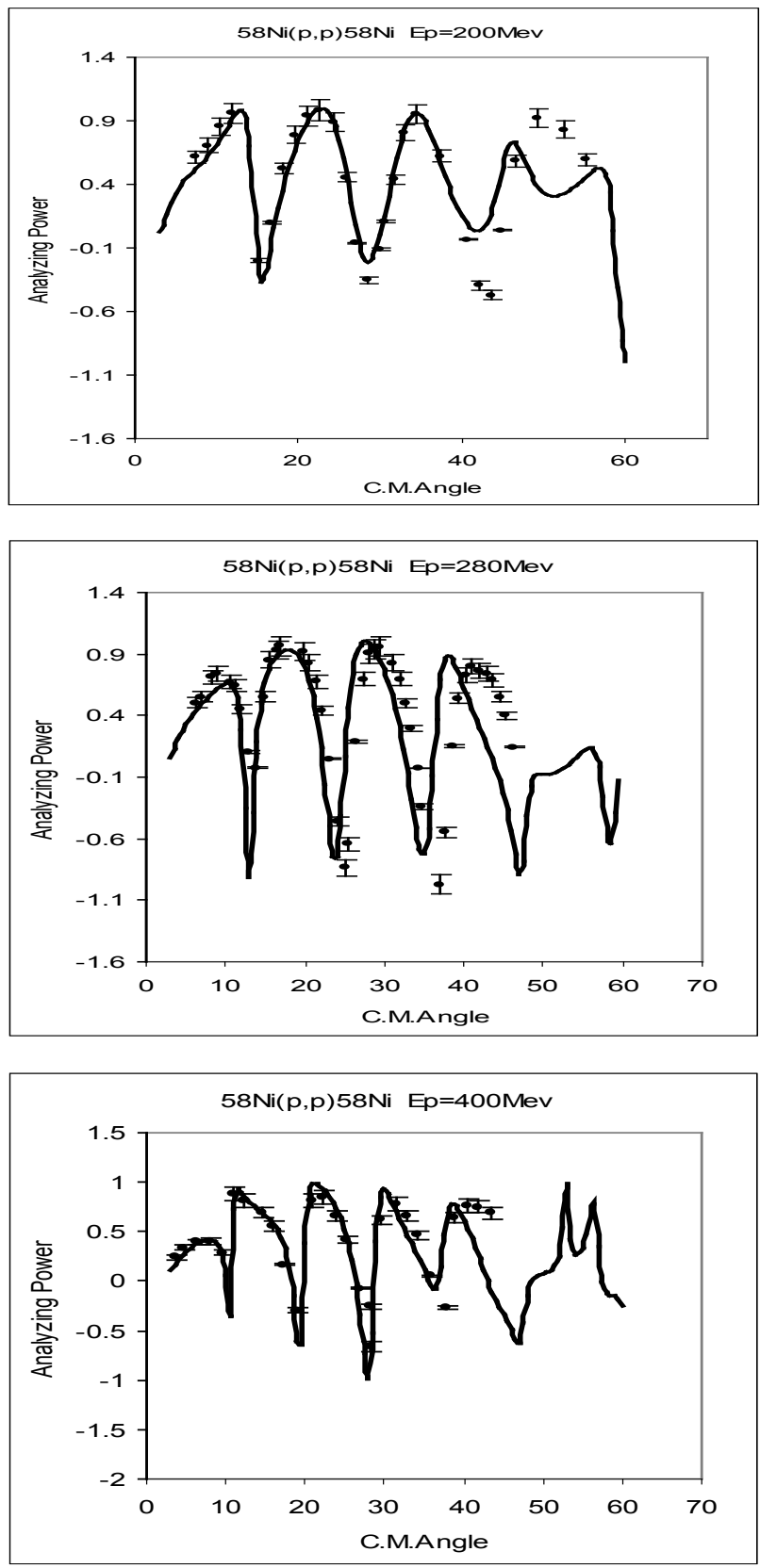

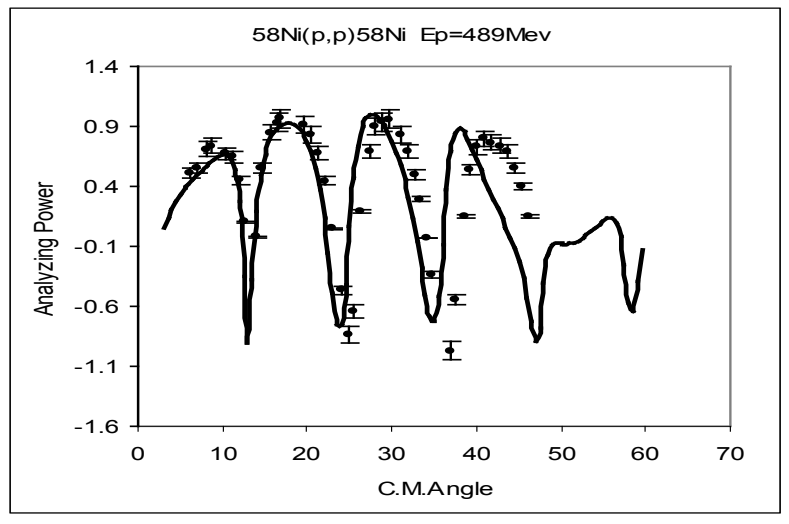

Figure 2. The analyzing power for $\mathrm{p}+58 \mathrm{Ni}$ elastic scattering at 200,280 , 400 and $489 \mathrm{MeV}$. The solid curves represent the global potential calculatins; the solid circles represent the experimental data with $8 \%$ error bar
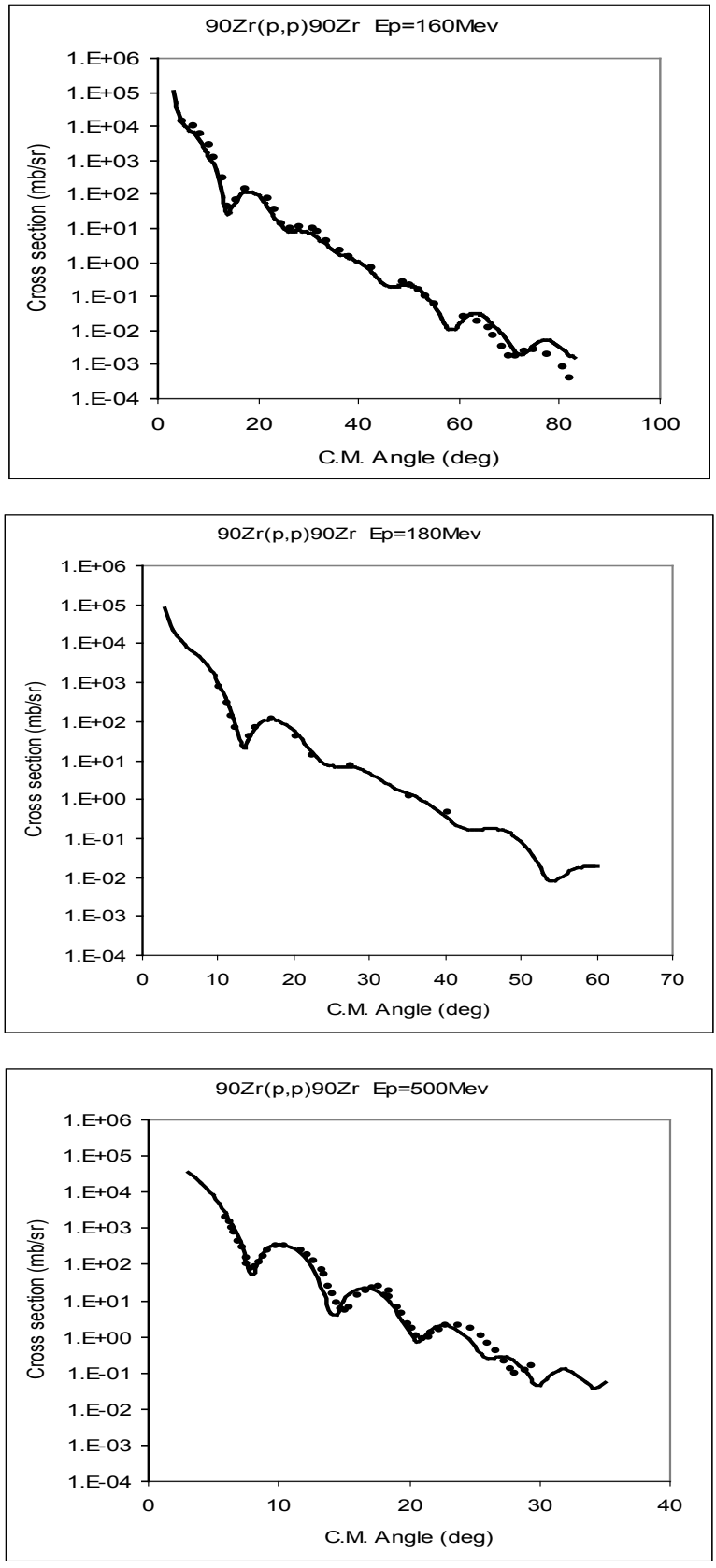


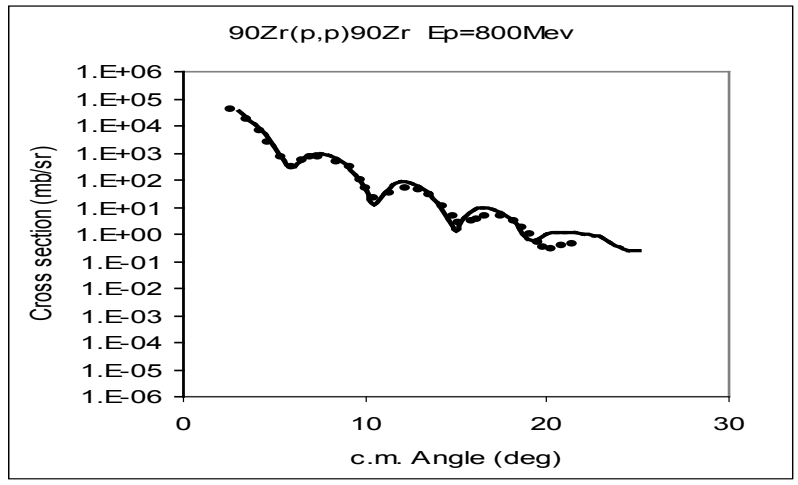

Figure 3. The differential cross section for $\mathrm{p}+90 \mathrm{Zr}$ elastic scattering at $160,180,500$ and $800 \mathrm{MeV}$. The solid curves represent the global potential calculations; the solid circles represent the experimental data
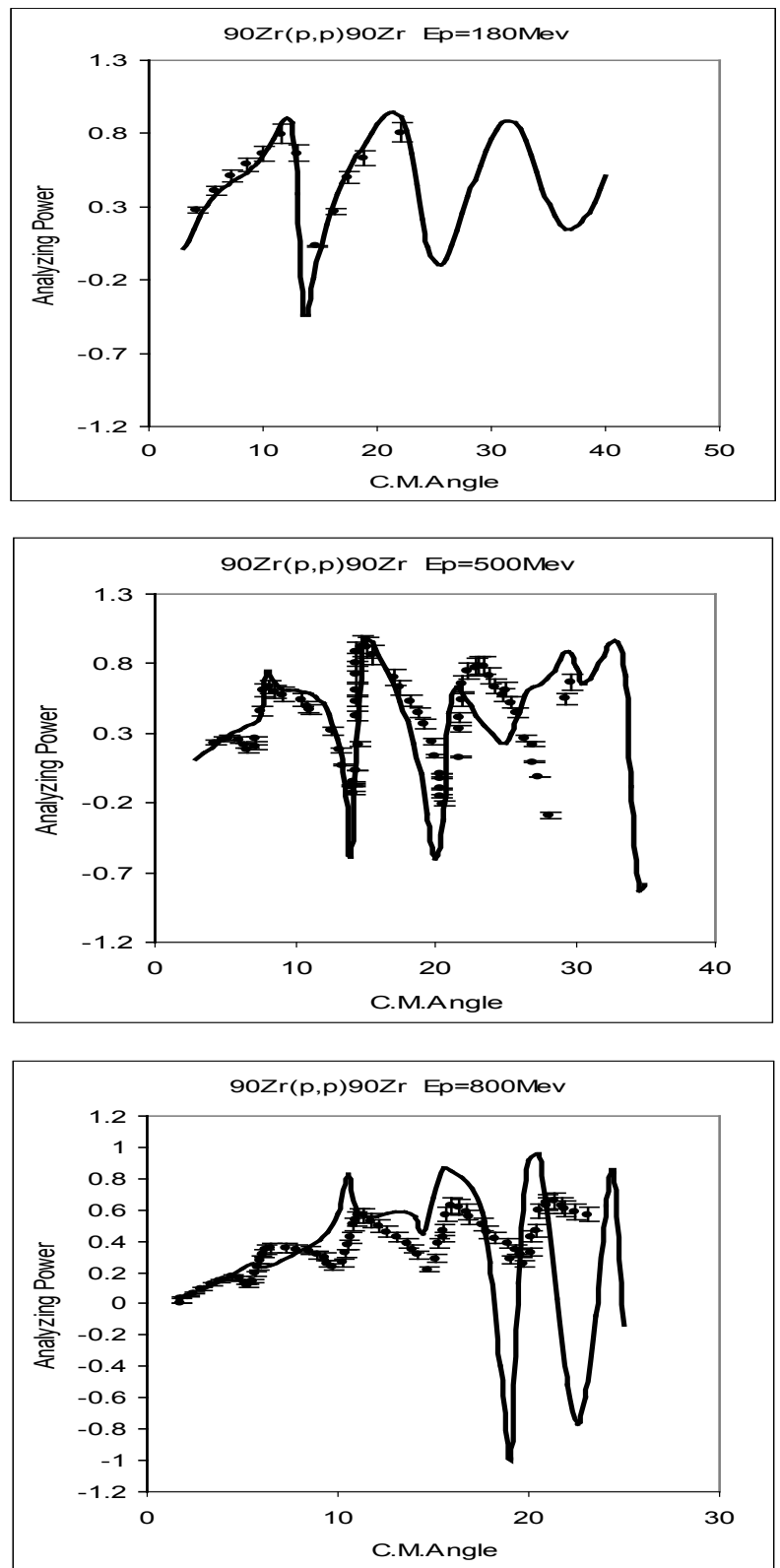

Figure 4. The analyzing power for $p+90 \mathrm{Zr}$ elastic scattering at 180,500 and $800 \mathrm{MeV}$. The solid curves represent the global potential calculations; the solid circles represent the experimental data with $8 \%$ error bar
In figures $(5-8)$, the real and imaginary potentials are shown to behave regularly with energy for both targets. Figures 9 and 11, show the real and imaginary central potentials for ${ }^{58} \mathrm{Ni}$ and ${ }^{90} \mathrm{Zr}$ respectively at different energies. We note that for the real part, the potentials are repulsive in the interior and attractive in the outer region. The imaginary central potentials all have similar shapes but with different depths. Figures 10 and 12, show the spin-orbit potentials energy dependence. In the surface region the potentials are close to each. The real spin-orbit potentials show negative pockets at short distances. The imaginary parts are similar to each other in the surface region showing a positive pocket at short distances. From the above we conclude that the differences between energies are more significant in the central part of their potentials than in the spin-orbit part. This is because the interior region of the nucleus plays a small role in proton- nucleus scattering at the energies and angles covered by this experiment compared to the surface region. At lower energies, the real central potential is always attractive and exhibits some structure inside the nucleus. For $58 \mathrm{Ni}$, at energies $>200 \mathrm{MeV}$, the real central potential has a repulsive core and becomes weakly attractive from about 3.7 $\mathrm{fm}$ onwards, reaching a minimum at about $4.7 \mathrm{fm}$. The same qualitative features occur for ${ }^{90} \mathrm{Zr}$. In the latter case the minimum in the potential is shifted to $5.4 \mathrm{fm}$. The imaginary central potential is more regular in shape and is always absorptive.
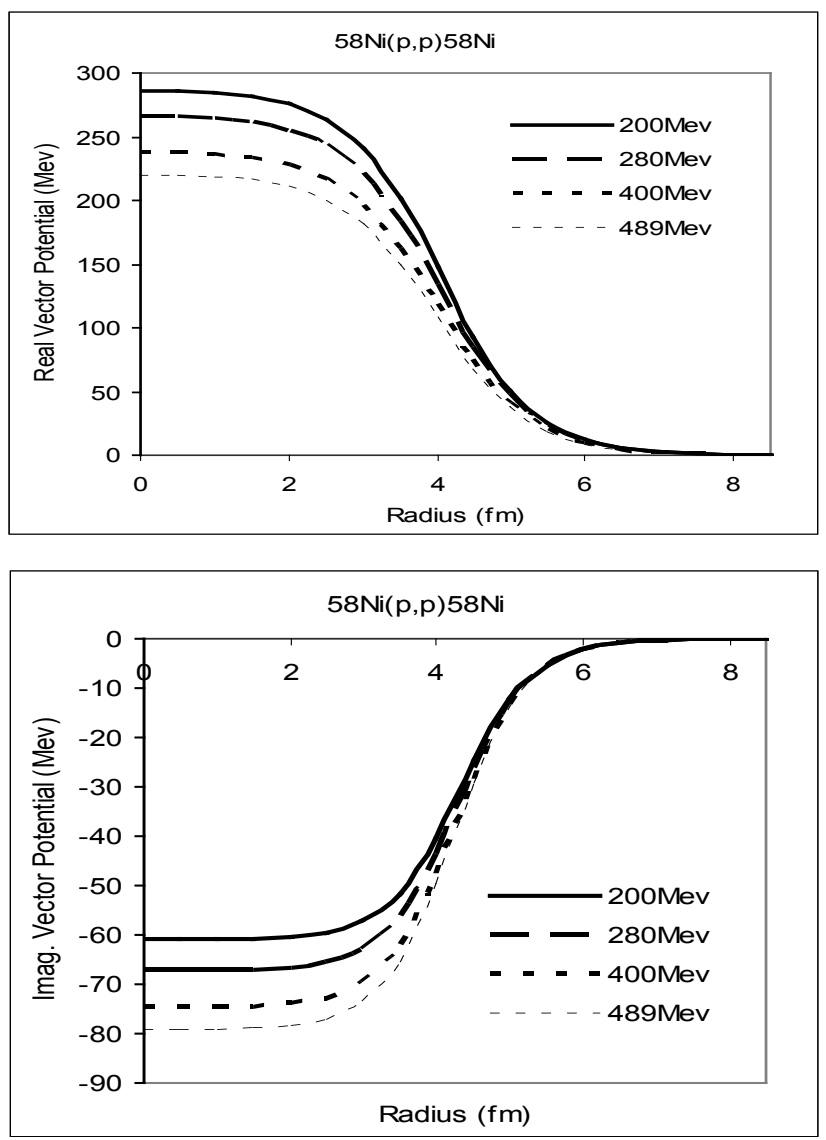

Figure 5. The real and imaginary vector optical potential for $\mathrm{p}+58 \mathrm{Ni}$ elastic scattering at 200, 280, 400 and $489 \mathrm{MeV}$ 

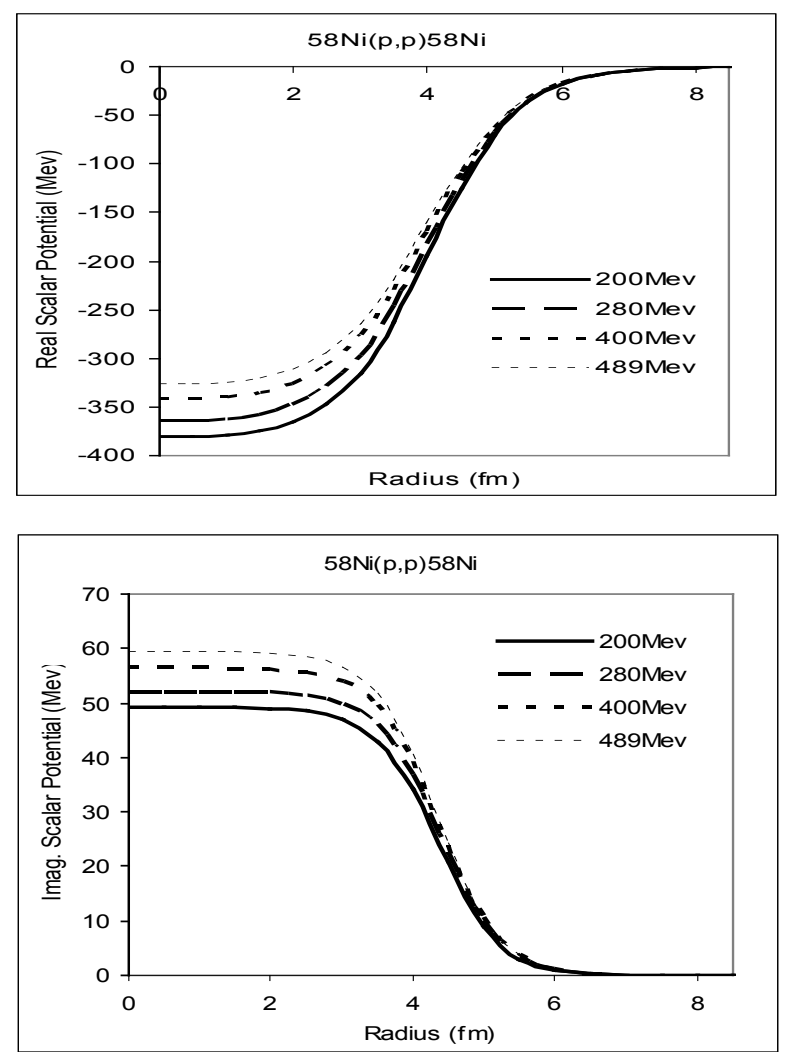

Figure 6. The real and imaginary scalar optical potential for $\mathrm{p}+58 \mathrm{Ni}$ elastic scattering at 200,280, 400 and $489 \mathrm{MeV}$
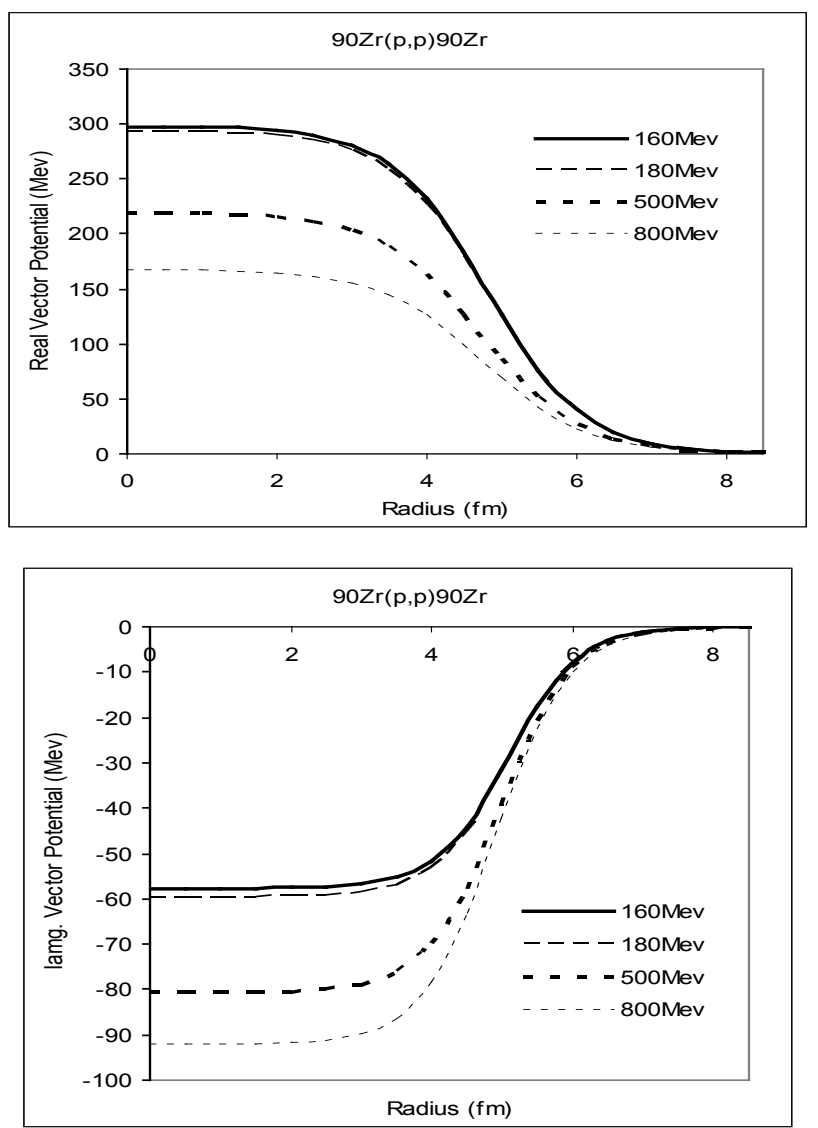

Figure 7. The real and imaginary vector optical potential for $\mathrm{p}+90 \mathrm{Zr}$ elastic scattering at 160, 180, 500 and $800 \mathrm{MeV}$
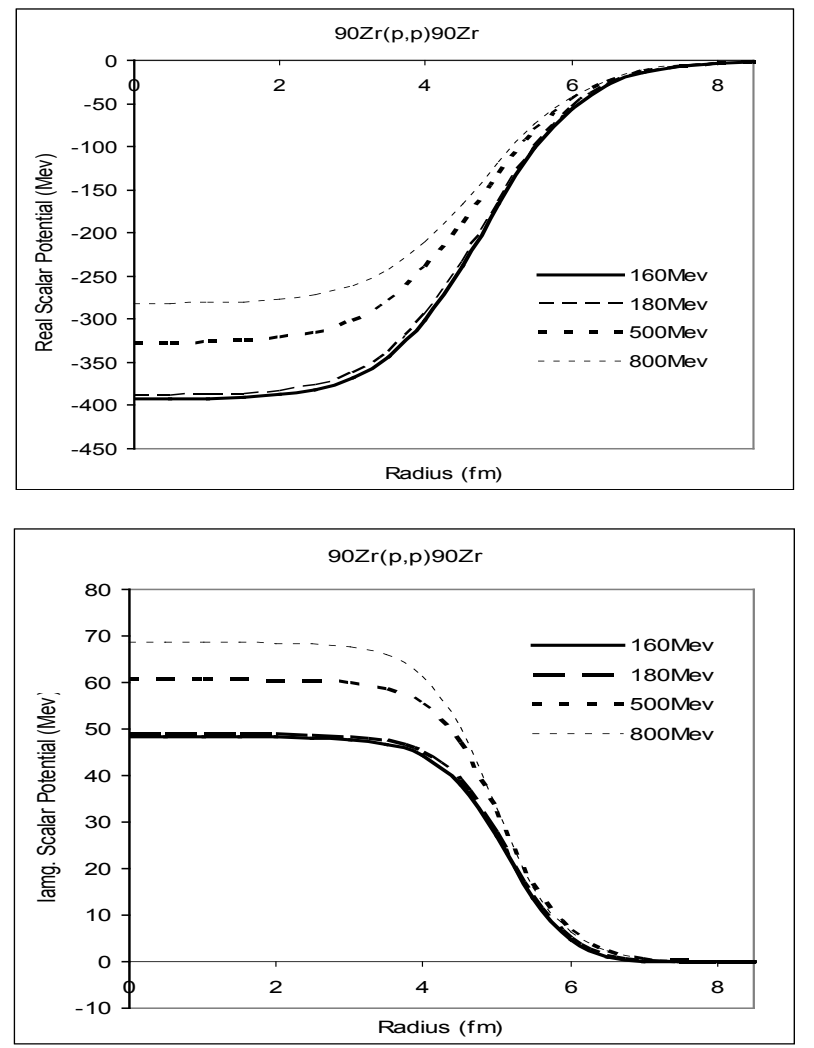

Figure 8. The real and imaginary scalar optical potential for $\mathrm{p}+90 \mathrm{Zr}$ elastic scattering at $160,180,500$ and $800 \mathrm{MeV}$
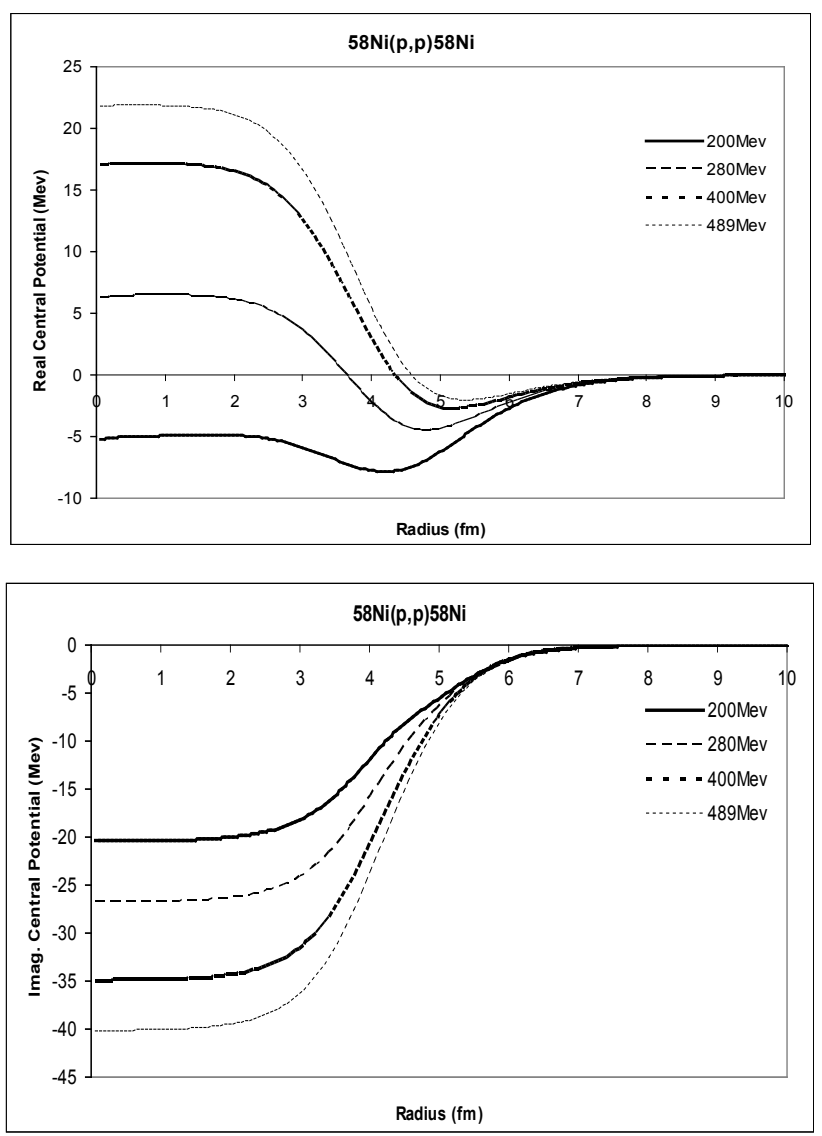

Figure 9. The real and imaginary central potentials for $\mathrm{Ni}$ at Different energies 


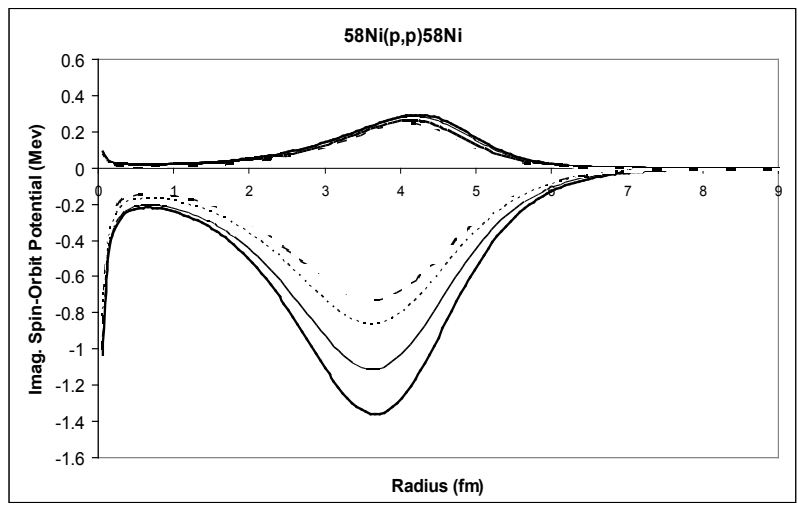

Figure 10. The real and imaginary spin-orbit potentials for Ni at Different energies
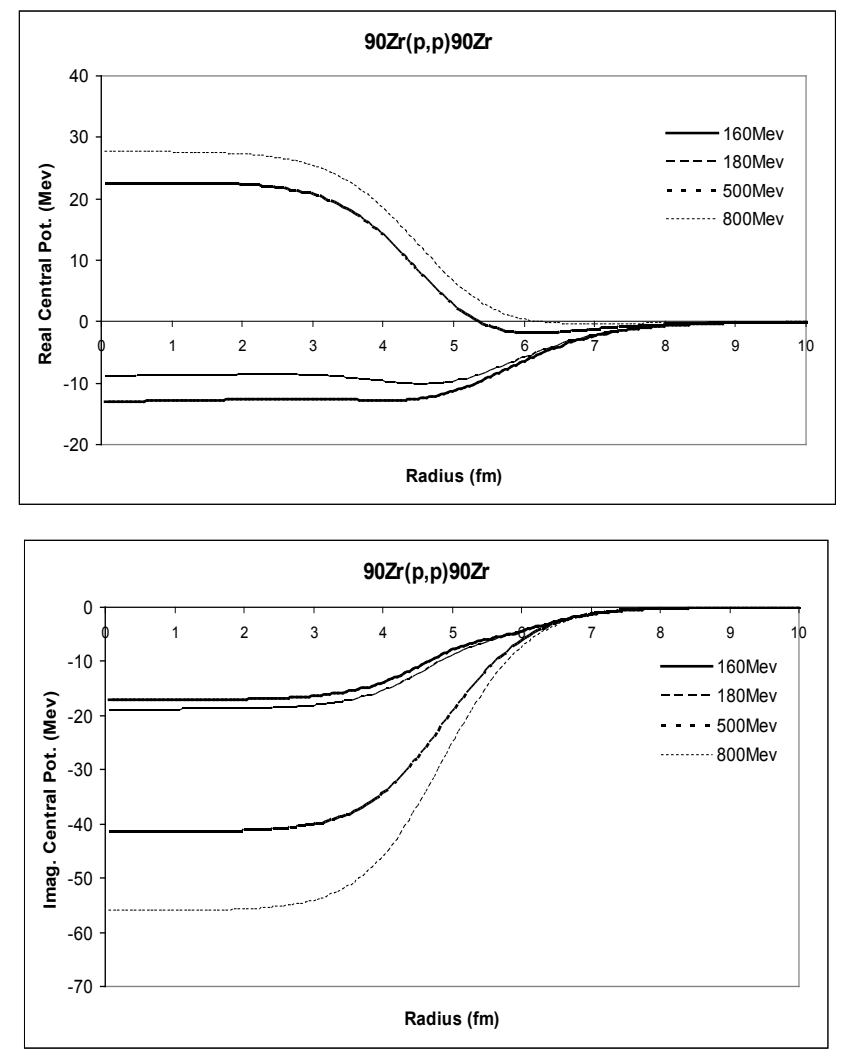

Figure 11. The real and imaginary central potentials for $\mathrm{Zr}$ at Different energies

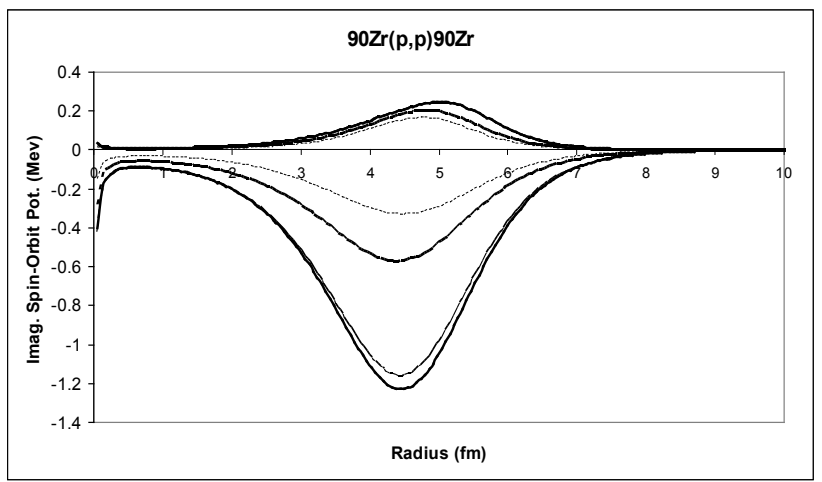

Figure 12. The real and imaginary spin-orbit potentials for $\mathrm{Zr}$ at Different energies

\section{Conclusions}

We have performed an extensive analysis of elastic proton scattering at intermediate energies from ${ }^{58} \mathrm{Ni}$ and ${ }^{90} \mathrm{Zr}$ nuclei within the global Dirac optical model. The value of this study is in the systematic of the results which allow some conclusions to be drawn. Firstly, the global fits to the differential cross sections are very good for both targets at all the energies. Secondly, we found the real vector and scalar potentials appear to be fairly well determined. They steadily decrease with energy and do not change much with the mass number. Thirdly, we found the behaviour of the real central effective potential shows the development of a "wine-bottle" shape in the transition energy region and the persistence of a small attractive potential in the nuclear surface region, even at 800 $\mathrm{MeV}$.

\section{REFERENCES}

[1] L.G. Arnold, B.C. Clark, R.L. Mercer and P. Schwandt, Phys. Rev. C23 (1981) 1949

[2] L.G. Arnold, B.C. Clark, E.D. Cooper, H.S. Sherif, D.A. Hutcheon, P. Kitching, J.M. Cameron, R.P. Liljestrand, R.N. MacDonald, W.J. McDonald, C.A. Miller, G.C. Neilson, W.C. Olsen, D.M. Sheppard, G.M. Stinson, D.K. McDaniels, J.R. Tinsley, R.L. Mercer, L.W. Swensen, P. Schwandt and C.E. Stronach, Phys. Rev. C25 (1982) 936

[3] B.C. Clark, S. Hama and R.L. Mercer, Proc. Conf. on interaction between medium energy nucleons in nuclei, 1982, ed. H.O. Meyer (AIP, New York, 1983) p. 260

[4] B.C. Clark, R.L. Mercer and P. Schwandt, Phys. Lett. 122B (1983) 211

[5] A.M. Kobos, E.D. Cooper, J.R. Rook and W. Haider, Nucl. Phys. A435 (1985) 677

[6] http://spot.colorado.edu/ kunz/DWBA.html

[7] S. Hama, B. C. Clark, E. D. Cooper, H. S. Sherif, and R. L. Mercer, Phys. Rev. C 41, (1990) 2737

[8] K. Kaki, H. Toki, Nucl. Phys. A696 (2001) 453

[9] Jerry . E.LISANTTI, Nucl. Phys. A529 (1991) 682

[10] A. Nadasen, P. Schwandt, P. P. Singh, W. W. Jacobs, A. D. Bacher, P. T. Debevec, M. D. Kaitchuck, and J. T. Meek, Phys. Rev. C23 (1981) 1023

[11] G.W. Hoffmann, L. Ray, M.L. Barlett, R. Fergerson, J. McGill and E.C. Milner Phys. Rev. Lett. 47 (1981) 1436.

[12] A. Rahbar, B. Aas, E. Bleszynski, M. Bleszynski, M. Haji-Saeid, G.J. Igo, F. Irom, G. Pauletta, A.T.M. Wang, J.B. McClelland, J.F. Amann, T.A. Carey, W.D. Cornelius, M. Barlett, G.W. Hoffmann, G. Glashausser, S. Nanda and M.M. Gazzaly, Phys. Rev. Lett. 47 (1981) 1811

[13] L. R, G.W. Hoffmann, G.S. Blampied, W.R. Coker and R.P. Liljestrand, Phys 